\title{
Epitaxial Structure Optimization for High Brightness InGaN Light Emitting Diodes by Using a Self-consistent Finite Element Method
}

\author{
Kyung-Soo Kim and Jong Chang Yi* \\ School of Electronic \& Electrical Engineering, Hongik University, 121-791 Seoul, Korea
}

(Received June 25, 2012 : revised July 31, 2012 : accepted August 1, 2012)

\begin{abstract}
The epitaxial layer structures for blue InGaN light emitting diodes have been optimized for high brightness applications with the output power levels exceeding $1000 \mathrm{~W} / \mathrm{cm}^{2}$ by using a self-consistent finite element method. The light-current-voltage relationship has been directly estimated from the multiband Hamiltonian for wurtzite crystals. To analyze the efficiency droop at high injection levels, the major nonradiative recombination processes and carrier spillover have also been taken into account. The wall-plug efficiency at high injection levels up to several thousand $\mathrm{A} / \mathrm{cm}^{2}$ has been successfully evaluated for various epilayer structures facilitating optimization of the epitaxial structures for desired output power levels.
\end{abstract}

Keywords: InGaN LEDs, Blue LEDs, FEM simulations, Self-consistent analysis

OCIS codes : (230.0230) Optical devices; (250.0250) Optoelectronics; (230.3670) Light-emitting diodes; (270.5580) Quantum electrodynamics

\section{INTRODUCTION}

High brightness nitride-based blue light emitting diodes (LEDs) have been intensively developed in the past ten years for indoor and outdoor displays and for illumination applications due to their potential advantages, such as high power efficiency and compactness in packaging [1-2]. Nevertheless, the power efficiency of LEDs, which is known as the primary advantage, should be further improved for solid-state lighting due to the efficiency droop at high injection levels [3-4]. Although the light-extraction efficiency has been significantly improved by many techniques, the internal quantum efficiency still suffers a major obstacle which results in efficiency droop behavior. Although the physical mechanisms behind the efficiency droop have not been fully established, the proposed major mechanisms at operating temperature include the nonradiative recombination processes and carrier spillover [3-4]. In this paper, a self-consistent light-current-voltage solver which takes all the major nonradiative recombination processes and current spillover into account has been utilized to investigate the power efficiency of LEDs with various epilayer structures at high injection levels. In the simulator, the radiative recombination rate is automatically estimated from the multiband Hamiltonian for the wurtzite crystals [5-9]. The non-radiative recombination processes such as Auger and Shockley-Read-Hall recombination processes have also been incorporated in the ambipolar current continuity equations [3,7]. The simulation results show the effects of epitaxial layer structure variations on the optical and electrical performance so that one can optimize the epitaxial structures such as the number of quantum wells, well widths and barrier widths with respect to the desired output power levels.

\section{SIMULATION ALGORITHM}

The Poisson equation is given by

$$
\nabla \cdot \varepsilon \nabla V=-\rho
$$

where $\varepsilon$ indicates the dielectric constant and the total charge $\rho$ can be represented as

$$
\rho=q\left(p_{i}-n_{i}-N_{A, i}{ }^{-}+N_{D, i}{ }^{+}\right)
$$

Here, $q$ is a positive quantity corresponding to the electron charge, and $p_{i}$ indicates the hole concentration at the $i$-th

\footnotetext{
*Corresponding author: wave@hongik.ac.kr

Color versions of one or more of the figures in this paper are available online.
} 
position node, $n_{i}$, the electron concentration, $N_{A i}{ }^{-}$, the acceptor ion concentration, $\mathrm{NDi}_{{ }^{+}}$, the donor ion concentration. For the self-consistent analysis, the Poisson equation can be rearranged as follows by using the Taylor series expansion;

$$
\begin{aligned}
& \nabla \cdot(\varepsilon \nabla V)+q\left(N_{D}-N_{A}-n+p\right)+q\left(N_{D}-N_{A}-n+p\right)^{\prime} . \\
& \left(V-V_{0}\right)=0
\end{aligned}
$$

where the terms inside the prime notation indicate the differential carrier concentrations at the given electrostatic potential, $V_{o}$. Then, the solution of electron and hole quasiFermi levels, $E_{f n}$ and $E_{f p}$ can be obtained from the current continuity equations as follow:

$$
\begin{aligned}
& q \frac{\partial n(i)}{\partial t}=\nabla \cdot J_{n}-q\left(R_{n}-G_{n}\right) \\
& q \frac{\partial p(i)}{\partial t}=-\nabla \cdot J_{p}-q\left(R_{p}-G_{p}\right)
\end{aligned}
$$

where $J_{n}$ and $J_{p}$ indicate the electron and hole current densities, respectively. $R_{n}$ and $G_{n}$ denote the recombination and generation rates for electrons and $R_{p}$ and $G_{p}$ for the holes in each layer. They include the Auger and SRH recombination processes [3] and spontaneous emission between the conduction band and valence band [7-9]. Basic semiconductor physics states that the drift and diffusion current densities can be expressed in terms of the quasi-Fermi levels as follows [10]:

$$
\begin{aligned}
& J_{n}=\mu_{n} n \nabla E_{f n}=\frac{\sigma_{n}}{q} \nabla E_{f n} \\
& J_{p}=\mu_{p} p \nabla E_{f p}=\frac{\sigma_{p}}{q} \nabla E_{f p}
\end{aligned}
$$

In the steady state, $\frac{\partial n(i)}{\partial t}=0$ and, $\frac{\partial p(i)}{\partial t}=0$ thus,

$$
\begin{aligned}
& \nabla \cdot J_{n}-q\left(R_{n}-G_{n}\right)=0 \\
& -\nabla \cdot J_{p}-q\left(R_{p}-G_{p}\right)=0
\end{aligned}
$$

Therefore,

$$
\begin{aligned}
& \nabla \cdot\left(\sigma_{n} \nabla E_{f n}\right)-q^{2}\left(R_{n}-G_{n}\right)=0 \\
& -\nabla \cdot\left(\sigma_{p} \nabla E_{f p}\right)-q^{2}\left(R_{n}-G_{n}\right)=0
\end{aligned}
$$

In this way, the quasi-Fermi levels $E_{f n}$ and $E_{f p}$ are resolved and they are used to evaluate the total charge densities given in Eq. (3). By iterating equations (2), (3), (10) and (11) until they satisfy both the Poisson equation and the continuity equations, one can obtain the self-consistent electrostatic potential and quasi-Fermi levels, which can be used to calculate the current densities given in Eq. (6) and (7) $[3,8]$.

The recombination and generation rates $R_{n}$ and $G_{n}$ in equations (10) and (11) include the band-to-band transition, Shockley-Read-Hall recombination, and the spontaneous emission rate as follow [3]:

$$
q\left(R_{n}-G_{n}\right)=B_{n r}\left(n p-n_{i}^{2}\right)+\frac{n p-n_{i}^{2}}{\tau_{p o}\left(n+n_{i}\right)+\tau_{n o}\left(p+n_{i}\right)}+\int R_{s p o n} d E
$$

Here, $B_{n r}$ indicates the non-radiative recombination rate, $\tau_{n o}$ the electron lifetime $\tau_{p o}$, the hole lifetime. $R_{\text {spon }}$ in the last term of equation (12) indicates the spontaneous emission rate between the conduction and the valence subbands, and it is directly calculated from the multi-band Hamiltonian equations including the strain and piezoelectric effects [5-9].

However, the analysis of the actual LEDs is not easy due to many unknown physical parameters such as the dislocation density, piezoelectric parameters, and deformation potential parameters. Even the commercial simulators usually show significant discrepancy between the simulation results and the experimental ones. In this paper, the material parameters were selected based on the references [5-6, 11]. Especially, the nonradiative recombination rate $B_{n r}$ usually increases as the carrier concentration increases due to the Auger process $[3,7]$ as follows:

$$
B_{n r}=B_{n r o}+C_{p} \times n
$$

Thus, as the LED power increases, the non-radiative recombination becomes more dominant. Hence the LED efficiency drops at high power regime, known as the efficiency

TABLE 1. Non-radiative recombination parameters used in the simulation

\begin{tabular}{c|c|c|c}
\hline \hline Parameter & Symbol & Value & Reference \\
\hline Band-to-band transition rate & $B_{n r o}$ & $4.78 \times 10^{-11} \mathrm{~cm}^{3} \mathrm{sec}^{-1}$ & {$[7]$} \\
\hline Auger recombination rate & $C_{p}$ & $1.5 \times 10^{-30} \mathrm{~cm}^{6} \mathrm{sec}^{-1}$ & {$[7]$} \\
\hline Electron capture time & $\tau_{n o}$ & $0.2 \mu \mathrm{s}$ & {$[3]$} \\
\hline Hole capture time & $\tau_{p o}$ & $0.2 \mu \mathrm{s}$ & {$[3]$} \\
\hline
\end{tabular}


droop. The relevant parameters used in this paper were listed in Table $1[3,7]$.

\section{SIMULATION RESULTS}

The epilayers of a typical commercial LED grown on sapphire substrate consist of $\mathrm{InGaN} / \mathrm{GaN}$ MQW active regions and the AlInGaN electron blocking layer. The epilayer structure under investigation in this work is as shown in Table 2 [12].

More precisely speaking, the MQW active regions consist of undoped 2.2-nm-thick $\mathrm{In}_{0.18} \mathrm{Ga}_{0.82} \mathrm{~N}$ wells and 6.6-nmthick $\operatorname{In}_{0.01} \mathrm{Ga}_{0.99} \mathrm{~N}$ barriers with varying doping concentrations of $5 \times 10^{18}, 3 \times 10^{18}, 2 \times 10^{18}, 1.26 \times 10^{18}$ and $1.0 \times 10^{18}$ $\left[\mathrm{cm}^{-3}\right]$, respectively. For an enhanced optical performance, p-doped $\mathrm{Al}_{0.2} \mathrm{In}_{0.07} \mathrm{Ga}_{0.73} \mathrm{~N}$ electron blocking layer (EBL) with a doping concentration of $7 \times 10^{18}\left[\mathrm{~cm}^{-3}\right]$ was added for suppression of electron overflow from the active layer to the p-doped cladding layer. $\mathrm{n}$-GaN is doped with $5 \times 10^{18} \mathrm{~cm}^{-3}$ and $\mathrm{p}-\mathrm{GaN}$ is doped with $3 \times 10^{19} \mathrm{~cm}^{-3}$. In the simulation, the buffer layer and substrate layer were excluded for convenience. The effect of the barrier doping was investigated and compared with the case of undoped barriers. In Table 2, the material number 1 denotes the quantum well $\left(\operatorname{In}_{0.18} \mathrm{Ga}_{0.82} \mathrm{~N}\right), 2$ the barrier $\left(\operatorname{In}_{0.01} \mathrm{Ga}_{0.99} \mathrm{~N}\right), 3$ the electron blocking layer $\left(\mathrm{Al}_{0.2} \mathrm{In}_{0.07} \mathrm{Ga}_{0.73} \mathrm{~N}\right)$ and 4 the cladding layer $\mathrm{p}-\mathrm{GaN}$ and $\mathrm{n}-\mathrm{GaN}$,

The barrier layers were divided artificially into two

TABLE 2. MQW LED epitaxial structure for the simulation

\begin{tabular}{c|c|c|c}
\hline \hline $\begin{array}{c}\text { Material } \\
\text { number }\end{array}$ & $\begin{array}{c}\text { Thickness } \\
(\mathrm{nm})\end{array}$ & $\begin{array}{c}\text { Nd doping } \\
\left(\mathrm{cm}^{-3}\right)\end{array}$ & $\begin{array}{c}\text { Na doping } \\
\left(\mathrm{cm}^{-3}\right)\end{array}$ \\
\hline 4 & 50 & $5.0 \times 10^{18}$ & 0 \\
\hline 2 & 3.3 & $5.0 \times 10^{18}$ & 0 \\
\hline 1 & 2.2 & 0 & 0 \\
\hline 2 & 6.6 & $\left(3.0 \times 10^{18}\right)$ & 0 \\
\hline 1 & 2.2 & 0 & 0 \\
\hline 2 & 6.6 & $\left(2.0 \times 10^{18}\right)$ & 0 \\
\hline 1 & 2.2 & 0 & 0 \\
\hline 2 & 6.6 & $\left(1.26 \times 10^{18}\right)$ & 0 \\
\hline 1 & 2.2 & 0 & 0 \\
\hline 2 & 6.6 & $\left(1.0 \times 10^{18}\right)$ & 0 \\
\hline 1 & 2.2 & 0 & 0 \\
\hline 3 & 3.3 & 0 & $7.0 \times 10^{18}$ \\
\hline 4 & 100 & 0 & $3.0 \times 10^{19}$ \\
\hline
\end{tabular}

1. well: $\mathrm{In}_{0.18} \mathrm{Ga}_{0.82} \mathrm{~N}$

2. barriers: $\operatorname{In}_{0.01} \mathrm{Ga}_{0.99} \mathrm{~N}$

3. electron blocking layer: $\mathrm{Al}_{0.2} \mathrm{In}_{0.07} \mathrm{Ga}_{0.73} \mathrm{~N}$

4. cladding layer: $\mathrm{GaN}$ regions and allocated to adjacent well regions for the simulation of the eigen energy states of each quantum well separately. The quasi-Fermi levels and electrostatic potential are resolved iteratively until the total current density converges within specified tolerance [5-9]. Fig. 1 shows the band diagram and current density characteristics of the InGaN LED with three quantum wells with undoped barriers. The barrier width is $6.6 \mathrm{~nm}$, well width $2.2 \mathrm{~nm}$ and electron blocking layer width $3.3 \mathrm{~nm}$.

Figure 1 (a) exhibits the band diagram and the quasi-Fermi levels for electrons and holes. The $\mathrm{n}$-contact is on the left and the p-contact is on the right side. One can clearly identify the quantum well regions although their conduction and valence bands are tilted due to the piezoelectric field. The electron blocking layer is located at the right side of the last quantum well layer. The Quasi-Fermi levels decrease as passing through each quantum well. This is due to the recombination in each quantum well. Fig. 1 (b) shows the electron and hole distributions in the active regions as well as in the EBL and cladding regions. Fig. 1 (c) shows the

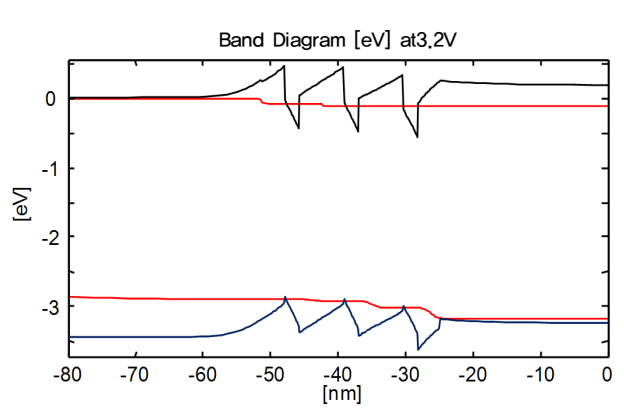

(a)

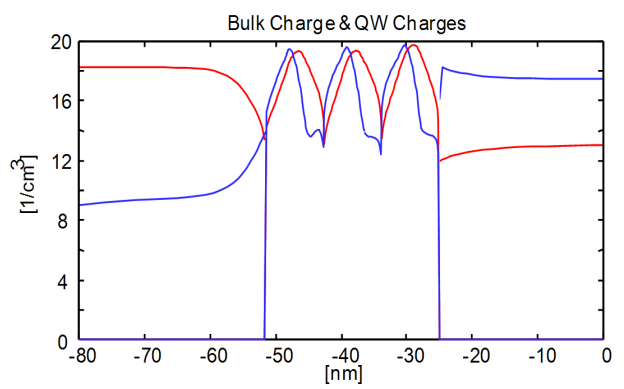

(b)

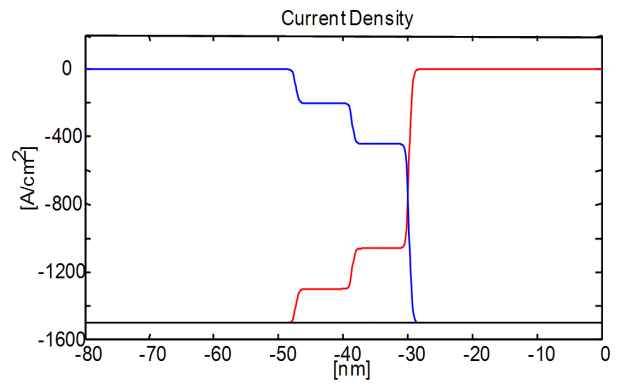

(c)

FIG. 1. (a) Band diagrams, (b) Charge profiles, and (c) Current densities of AlInGaN LED with three quantum wells with undoped barriers. 


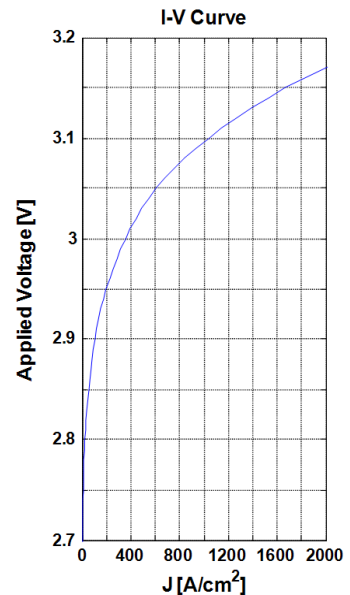

(a)

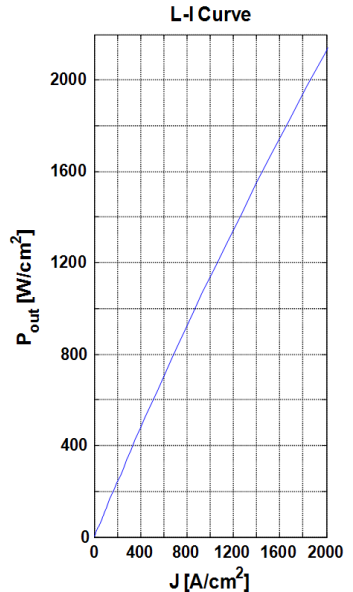

(b)

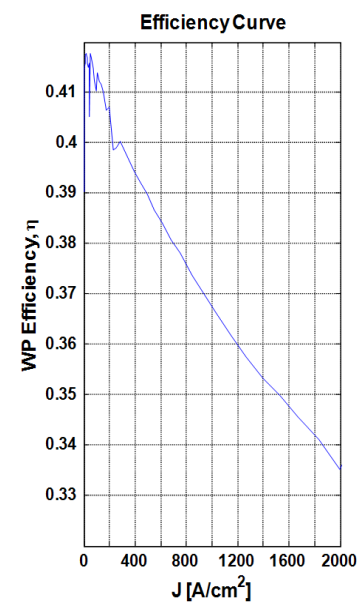

(c)

FIG. 2. (a) Electrical I-V (b) Optical L-I, and (c) WP efficiency characteristics of the InGaN MQW LED.

electron and hole current densities. The sum of the electron and hole current densities is obviously constant.

Figure 2 shows the resulting electrical and optical characteristics. The current-voltage characteristics is shown in Fig. 2 (a), where one can see that the turn-on voltage is about 2.8 V. Fig. 2 (b) shows the light output characteristics of the InGaN LED with three quantum wells at the applied voltage between 2.7 and $3.2 \mathrm{~V}$. The optical performance is evaluated by wall plug (WP) efficiency by assuming unity extraction efficiency. The conventional optical extraction efficiency is around $70 \%$, [13] and there are many new technologies to improve this area. Thus, the actual WP efficiency should be more or less $70 \%$ of the value estimated from this simulator. Nevertheless, the WP efficiency is more practical quantity than the internal quantum efficiency since it quantifies the efficiency of conversion of electrical energy into an emitted external optical energy. When the applied voltage varies from $2.7 \mathrm{~V}$ to $3.2 \mathrm{~V}$, the optical power density rises above $2,000\left[\mathrm{~W} / \mathrm{cm}^{2}\right]$ and current density above 2,000 $\left[\mathrm{A} / \mathrm{cm}^{2}\right]$. Optical power density versus current density is linear until $1,200\left[\mathrm{~W} / \mathrm{cm}^{2}\right]$ and becomes nonlinear beyond

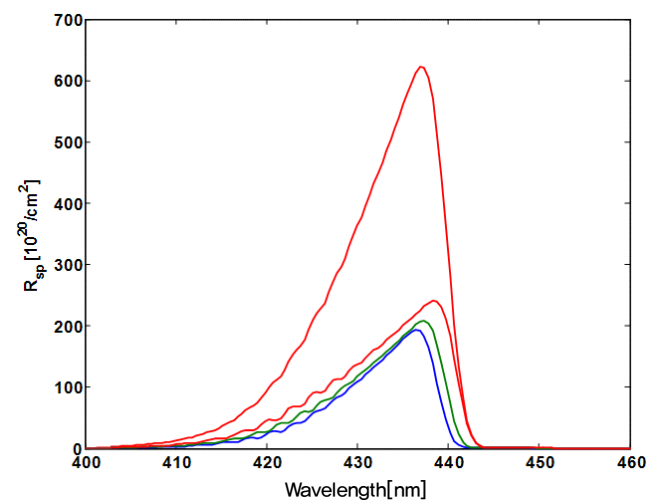

FIG. 3. Optical emission spectra of InGaN LED with three quantum wells.

thereafter as apparent in Fig. 2 (b). As apparent in Fig. 2 (c), WP efficiency is reduced as current density increases. WP efficiency is approximately 0.365 at $1,000\left[\mathrm{~A} / \mathrm{cm}^{2}\right]$ and 0.335 at $2,000\left[\mathrm{~A} / \mathrm{cm}^{2}\right]$.

Figure 3 shows three optical emission spectra from each of the quantum wells when the applied voltage is $3.2 \mathrm{~V}$. Each of the emission spectra is obtained by integrating the spontaneous emission rate over each of the quantum well regions. The top curve represents the sum of the three emission spectra from the three quantum wells, and the peak wavelength is $437 \mathrm{~nm}$ at the given applied voltage. The peak wavelength gradually shifted to the shorter wavelength as the applied voltage increased.

\section{EPITAXIAL STRUCTURE OPTIMIZATION}

Figure 4 shows the variation of the WP efficiency as the number of quantum wells varies in the case of $2.2 \mathrm{~nm}$ wide quantum wells, $6.6 \mathrm{~nm}$ wide barriers, and $3.3 \mathrm{~nm}$ wide electron blocking layer. The WP efficiency decreases as the optical power density increases due to the higher Auger recombination as shown in Fig. 4. It shows the variation of the WP efficiency at the optical power densities of $500,1,000,1,500$ and $2,000\left[\mathrm{~W} / \mathrm{cm}^{2}\right]$, respectively. From Fig. 4, one can see that the WP efficiency becomes the maximum when the number of wells is three. The maximum WP Efficiency is 0.394, 0.374, 0.359 and 0.342 for the cases of optical output power densities at 500, 1,000, 1,500 and $2,000\left[\mathrm{~W} / \mathrm{cm}^{2}\right]$, respectively. Again, the actual WP efficiency would be $70 \%$ of the estimated ones due to the non-unity optical extraction efficiency.

Figure 4 clearly shows that there is an optimum number of quantum wells with respect to the WP efficiency. The optimum number is determined by compromising the carrier feeding which is more efficient at fewer quantum wells and the nonradiative recombination which becomes more significant at higher carrier concentration inevitably occurring in LEDs with fewer quantum wells. Thus, the optimum number of quantum wells exists and will vary depending 
on the actual epilayer structures.

Figure 5 shows the effect of barrier width on the WP efficiency in the case of three $2.2 \mathrm{~nm}$-wide quantum wells and a $3.3 \mathrm{~nm}$ wide electron blocking layer. One can see that the WP efficiency becomes the maximum when the barrier width is near $6.6 \mathrm{~nm}$. The WP efficiency peaks at 0.394 for $500 \mathrm{~W} / \mathrm{cm}^{2}$ light output density, and 0.374 for $1,000 \mathrm{~W} / \mathrm{cm}^{2}$ light output density. WP efficiency increases smoothly from $5.6 \mathrm{~nm}$ to $6.6 \mathrm{~nm}$, but it decreases rapidly beyond $6.6 \mathrm{~nm}$ and below $5.2 \mathrm{~nm}$. Therefore, one can see that the optimum barrier width is near $6.6 \mathrm{~nm}$ for the given quantum well structures.

Figure 5 clearly shows that there is an optimum value of barrier width with respect to the WP efficiency. The optimum value is determined by compromising the carrier feeding which is more efficient at smaller barrier width and the quantum confinement effect which becomes more significant at larger barrier width. Thus, the optimum value of barrier width exists and will vary depending on the actual epilayer structures.

Figure 6 shows the effect of electron blocking layer width on the WP efficiency for the case of three $2.2 \mathrm{~nm}$ wide quantum wells and $6.6 \mathrm{~nm}$ wide barriers. From Fig.6, one can see that the WP efficiency does not vary signifi-

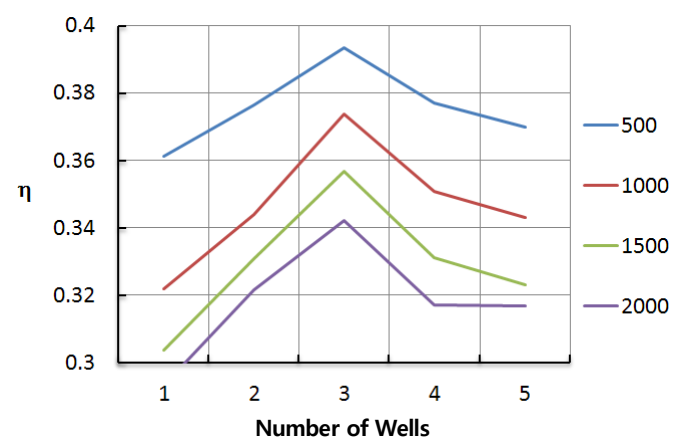

FIG. 4. The variation of the WP efficiency vs. the number of quantum wells. The labels indicate the light output power densities $\left[\mathrm{W} / \mathrm{cm}^{2}\right]$.

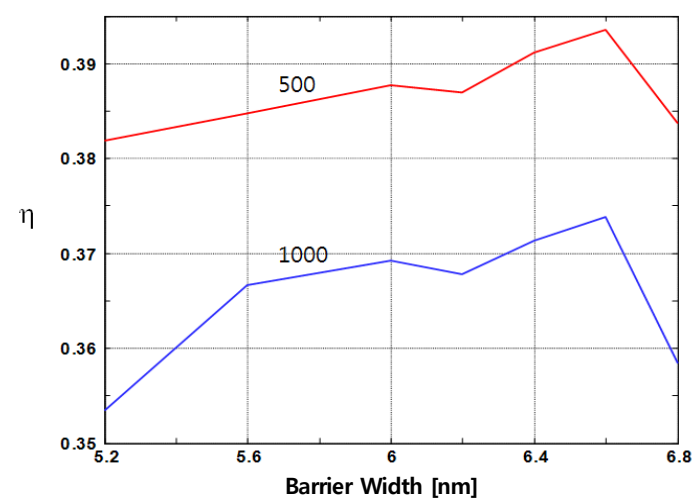

FIG. 5. The variation of the WP efficiency vs. the barrier width $[\mathrm{nm}]$. The labels indicate the light output power densities $\left[\mathrm{W} / \mathrm{cm}^{2}\right]$. cantly as long as the electron blocking layer width is moderately wide between $2.2 \mathrm{~nm}$ and $6.3 \mathrm{~nm}$. When the electron blocking layer width is, however, smaller than 2.2 $\mathrm{nm}$, or greater than $6.3 \mathrm{~nm}$, the WP efficiency drops abruptly. Therefore, one needs to keep the EBL thickness within this range.

Figure 7 shows the effect of doping in the barrier regions on the WP efficiency for the same epilayer structures. For the doped structures, the doping concentration in each of the barrier regions is as shown in the parenthesis in Table 2. The WP efficiencies were estimated for the optical power density of $500 \mathrm{~W} / \mathrm{cm}^{2}$. From Fig. 7, one can see that the WP efficiencies of the MQW LEDs with doped barriers are worse than those of the undoped structures. Therefore, one should take an extreme care to utilize the doped barriers in the MQW structures because such intentional doping can deteriorate the light output performance of the LEDs.

Figure 8 shows the variation of the WP efficiency as the quantum well width varies. When the quantum well width is modified, the material composition is also

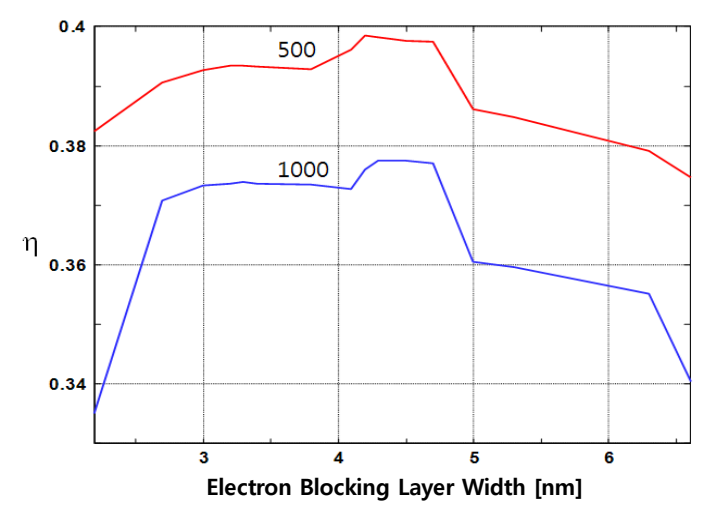

FIG. 6. The variation of the WP efficiency vs. the electron blocking layer width [nm]. The labels indicate the light output power densities $\left[\mathrm{W} / \mathrm{cm}^{2}\right]$.

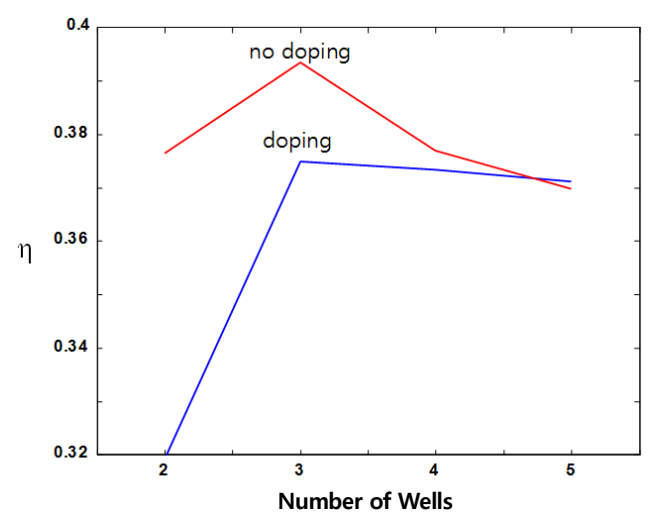

FIG. 7. The variation of the WP efficiency vs. the number of quantum wells. The labels indicate whether the barrier regions are doped or not. The light output power density is $500\left[\mathrm{~W} / \mathrm{cm}^{2}\right]$. 


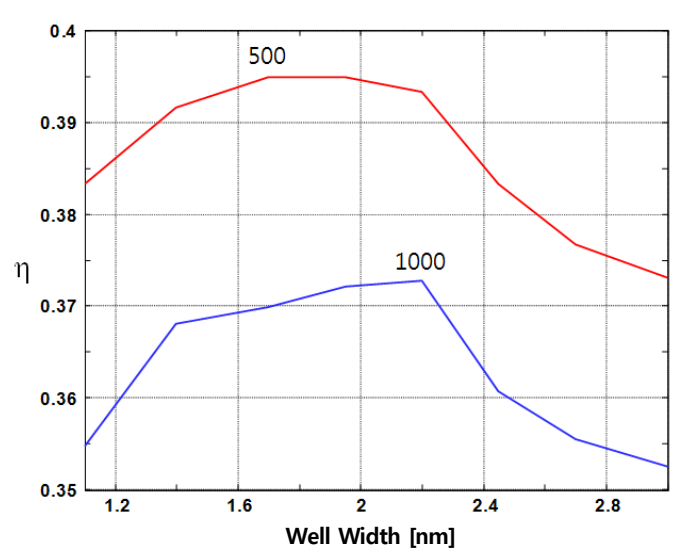

FIG. 8. The variation of the WP efficiency vs. the quantum well width. The labels indicate the light output power densities $\left[\mathrm{W} / \mathrm{cm}^{2}\right]$.

modified accordingly to maintain the peak wavelength near $440 \mathrm{~nm}$. Again the barriers are undoped and their widths are kept at $6.6 \mathrm{~nm}$ along with the electron blocking layer width at $3.3 \mathrm{~nm}$. From Fig. 8, one can see that the highest efficiency is produced when the well width is $1.7 \mathrm{~nm}$ where the efficiency is 0.395 for the output power of 500 $\left[\mathrm{W} / \mathrm{cm}^{2}\right]$. However, for the output power of $1,000\left[\mathrm{~W} / \mathrm{cm}^{2}\right]$, the WP efficiency becomes maximum when the well width is $2.2 \mathrm{~nm}$. When the well width is below $1.4 \mathrm{~nm}$ or above $3.0 \mathrm{~nm}$, the efficiency decreased abruptly. Therefore, one can conclude that well width near $2.2 \mathrm{~nm}$ is optimum for high-brightness application with power levels exceeding $1,000 \mathrm{~W} / \mathrm{cm}^{2}$ for the given epilayer structure.

Figure 8 clearly shows that there is an optimum value of quantum well width with respect to the WP efficiency. The optimum value is determined by compromising the quantum confinement effect which becomes more significant at smaller well width and Auger recombination which becomes more significant at higher carrier concentration inevitably occurring in narrower well width. Thus, the optimum value of quantum well width exists and will vary depending on the actual epilayer structures.

\section{CONCLUSION}

In this paper, the device performance of the AlInGaN blue LEDs on various epilayer structures has been investigated by using a self-consistent FEM simulator which can evaluate the overall light output and the injection current at a given bias voltage. The feasibility of the simulator has been demonstrated by applying to various epitaxial structures. By investigating the L-I-V characteristics of the blue LEDs regarding the WP efficiency for desired power output levels, the epilayer structures have been optimized with respect to the number of quantum wells, the widths of the wells, the barriers, and the electron blocking layer, as well as the doping levels. The simulation results indicates that the maximum WP efficiency can be as high as $28 \%$ and $26 \%$ for a high intensity LED for a power output level of 500 and $1,000 \mathrm{~W} / \mathrm{cm}^{2}$, respectively, assuming that the extraction efficiency is around $70 \%$. Such WP efficiency can be further enhanced by optimizing the barrier materials or the substrate crystal orientations. One last step before deploying this simulator to an actual epilayer growth optimization, however, would be the selection of the right set of the material parameters which vary significantly from batch to batch. After such procedures, this simulator can be effectively utilized to optimize complicate epilayer structures for highbrightness LEDs on wurtzite material systems.

\section{ACKNOWLEDGMENT}

This work was supported by National Research Foundation of Korea (NRF-2011-002-7208).

\section{REFERENCES}

1. C.-F. Chu, C.-C. Cheng, W.-H. Liu, J.-Y. Chu, F.-H. Fan, H.-C. Cheng, T. Doan, and C. A. Tran, "High brightness GaN vertical light-emitting diodes on metal alloy for general lighting application," Proc. IEEE 98, 1197-1207 (2010).

2. Y.-J. Lee, T.-C. Lu, H.-C. Kuo, and S.-C. Wang, "High brightness GaN-based light-emitting diodes," Journal of Display Technology 3, 118-125 (2007).

3. U. Ozgur, H. Liu, X. Li, X. Ni, and H. Morkoc, "GaN-based light-emitting diodes: efficiency at high injection levels," Proc. IEEE 98, 1180-11196 (2010).

4. C. H. Wang, J. R. Chen, C. H. Chiu, H. C. Kuo, Y.-L. Li, T. C. Lu, and S. C. Wang, "Temperature-dependent electroluminescence efficiency in blue InGaN-GaN light-emitting diodes with different well widths," IEEE Photon. Technol. Lett. 2, 236-238 (2010).

5. J. Piprek, Semiconductor Optoelectronic Devices (Academic Press, UCSB, USA, 2003), Chapter 3.

6. J. Piprek (ed.), Nitride Semiconductor Devices-principles and Simulation (Wiley-VCH, Weinheim, Germany, 2007), Chapter 1-5.

7. A. Tomczyk, R. P. Sarzala, T. Czyszanowski, M. Wasiak, and W. Nakwaski, "Fully self-consistent three-dimensional model of edge-emitting nitride diode lasers," Opto-electronics Review 11, 65-75 (2003).

8. D. W. Lim, H. C. Cho, H. K. Sung, and J. C. Yi, "A PSPICE circuit modeling of strained AlGaInN laser diode based on the multilevel rate equations," J. Opt. Soc. Korea 13, 386-391 (2009).

9. Y. Chung, J. C. Yi, and H. S. Cho, "Modeling of active layer and injection-locking characteristics in polarized and unpolarized Fabry-Perot laser diodes," Korean J. Opt. Photon. 23, 42-51 (2012).

10. B. G. Streetman and S. K. Banerjee, Solid State Electronic Devices, 6th ed (Pearson Prentice Hall, New Jersey, USA, 2006), Chapter 4.

11. I. Vurgaftman and J. R. Meyer, "Band parameters for 
nitride-containing semiconductors," J. Appl. Phys. 94, 3675 -3696 (2003).

12. S. J. Leem, Y. C. Shin, E. H. Kim, C. M. Kim, B. G. Lee, Y. Moon, I. H. Lee, and T. G. Kim, "Optimization of InGaN/GaN multiple quantum well layers by a two-step varied-barrier-growth temperature method," Semiconductor Science and Technology 23, 125039 (2008).

13. S. J. Lee, "Difference in design consideration between InGaN and conventional high-brightness light-emitting diodes," J. Opt. Soc. Korea 2, 13-21 (1998). 